\title{
Behavioral Impact of Drivers' Roles in Automated Driving
}

\author{
Bryan Reimer ${ }^{1}$, \\ Anthony Pettinato', \\ Lex Fridman ${ }^{3}$, Joonbum Lee ${ }^{4}$, \\ Bruce Mehler 5 \\ MIT AgeLab \& New England \\ Univ. Transportation Center \\ Cambridge, MA, USA \\ reimer@mit.edu ${ }^{1}$, apett@mit.edu ${ }^{2}$, fridman@mit.edu ${ }^{3}$,joonbum@mit.edu ${ }^{4}$, bmehler@mit.edu ${ }^{5}$, \\ bseppelt@touchstoneevaluations.com ${ }^{6}$,junghee@mit.edu ${ }^{7}, \mathrm{kdi} @$ mit.edu $^{8}$
}

\begin{abstract}
This study explores the effects of minor changes in automation level on drivers' engagement in secondary activities. Three levels of automation were tested: manual, semi-autonomous, and fully-autonomous. Potential distractor items were present and participants were instructed they could use them if they felt it was safe. Hand positions and engagement in secondary activities were manually coded. Participants were significantly less likely to engage in a secondary activity in semi-autonomous than fully-autonomous mode. Likewise, they were significantly less likely to use two hands to interact with a secondary activity in semi-autonomous mode than fully-autonomous mode. Gaze classification for each of the driver roles revealed that increasing levels of automation resulted in an increasing percentage of off-road glance durations. These observations suggest that in the event of automation failures, a driver in semi-autonomous driving may be in a somewhat better position to retake control and avoid collisions than during fully autonomous driving.
\end{abstract}

\section{Author Keywords}

Driver distraction; Autonomous driving; Semi-autonomous driving; Driver behavior; Automated gaze annotation; Human Factors

\section{ACM Classification Keywords}

H.5.m. Information interfaces and presentation (e.g., HCI): Miscellaneous; J.7. Computers in other systems.

\section{INTRODUCTION}

Vehicle automation promises benefits for the individual driver through to societal-level impacts, including, among

Permission to make digital or hard copies of all or part of this work for personal or classroom use is granted without fee provided that copies are not made or distributed for profit or commercial advantage and that copies bear this notice and the full citation on the first page. Copyrights for components of this work owned by others than the author(s) must be honored. Abstracting with credit is permitted. To copy otherwise, or republish, to post on servers or to redistribute to lists, requires prior specific permission and/or a fee. Request permissions from Permissions@acm.org.

Automotive'UI 16, October 24 - 26, 2016, Ann Arbor, MI, USA

Copyright is held by the owner/author(s). Publication rights licensed to ACM.

ACM 978-1-4503-4533-0/16/10 ..\$15.00

DOI: http://dx.doi.org/10.1145/3003715.3005411. others, a reduced number and severity of crashes, increased mobility options, reduced driving demands (potentially freeing time for drivers to engage in work-related or entertainment activities), eased traffic congestion, transport for non-driving populations, and an overall reduction in pollution from vehicle emissions [20,26]. At the individual driver level, use of vehicle automation has already demonstrated benefits with noted improvements in performance in routine driving conditions. In maintaining a set headway to a lead vehicle, use of early adaptive cruise control (ACC) systems on motorways resulted in a net positive benefit over manual control with a relative reduction in the rate of harsh braking events, time headways below $0.5 \mathrm{~s}$, and incidents [13]. More recently, the deceleration ACC initiates in response to a slowing lead vehicle has been shown to help direct driver attention to the road [30]. ACC use however, may impact visual attention in ways that are not yet fully understood and reported levels of trust in the system have been found, in one sample of new users, to be higher than what would be suggested by objective measures of their physiological arousal [21]. In the latter instance, participants who were provided with an opportunity to become familiar with the operation of an ACC system reported relatively high levels of trust in the system after the drive, but showed higher levels of heart rate and skin conductance when $\mathrm{ACC}$ was on vs. when it was off.

While use of semi-automated technologies like ACC (which maintains a set following distance to a lead vehicle) or lane-keeping assist (LKA; which helps to maintain the vehicle's position within the lane) can help to maintain or improve safety margins from those a driver may him/herself adopt, these technologies are not perfect (i.e. there are situations where current sensors are expected to fail) and functionally require supervision. More advanced, semiautomated driving technologies (e.g., Autopilot) build-upon the basic functionalities of ACC and LKA with added automation to maintain the vehicle's position in the center of the lane and, in some instances, follow the trajectory of a lead vehicle. Semi-automated driving technologies in deployment are currently limited in the amount of force they apply to steer, and to accelerate or decelerate the 
vehicle, and in their sensing capability to detect lane lines, leading vehicles and the presence of events or objects in or near-to the road that may pose a collision threat. Because of these limitations, these technologies still require a level of driver supervision to ensure safe driving performance. At the operational level, the degree to which the driver needs to supervise different levels of semi-automated driving technology may differ but, functionally, today the driver remains legally responsible for vehicle control.

In semi-autonomous driving, there is an expectation that the driver will monitor the driving environment and provide fallback driving control (SAE level 2) [22], or to provide fallback driving performance when requested to intervene without being required to monitor the driving environment (SAE Level 3) [22]. In fully-autonomous driving, the driver is not responsible for safe operation of the vehicle and is not expected to respond to any requests to intervene (SAE Level 4) [22]. What distinguishes semi-automated from fully-autonomous driving is the expectation that the driver monitor the driving environment - to act as a supervisor of the automated system(s).

This fundamental shift in a driver's role from a controller during manual operation of the vehicle to a supervisor during semi-autonomous driving poses some key human factors concerns related to monitoring and task management costs $[25,28,32]$. Being removed from moment-to-moment vehicle control tasks, drivers experience a loss of critical haptic, auditory, and visual cues that inform them of the vehicle's response to roadway and environment conditions; no longer receiving this information at the same frequency, drivers may develop misconceptions about the automation's status and behavior relative to its interaction with the driving environment, which can lead to improper use of the technology (e.g. over trust, mode confusion, etc.) $[10,17,23,25,29]$. Of further concern, the monitoring roles contain many elements of a vigilance task, and humans are notably ineffective in the requirement to maintain sustained attention to infrequent events [12,27,31]. Many, if not all, of the situations for which a driver would be requested to intervene are likely to be unexpected, rare, or both. It is the need to keep drivers "in-the-loop" in the event of a safety-critical event that either goes undetected or to which the vehicle automation is unable to adequately respond to, thus requiring the driver to take-over manual control, that is of particular concern.

Recent research confirms that in being relieved of the moment-to-moment control task requirements, drivers using semi-autonomous systems are inclined to direct their attention away from the forward roadway to other locations $[9,14,24]$. In a recent study looking at driver's willingness to engage in non-driving-related activities during highly automated vehicle control (ACC + lane keeping), drivers became more heavily involved with in-vehicle entertainment activities compared to manual driving, and were consequently less attentive to the road ahead [9]. The resulting reduced awareness of the situation translates into difficulty responding to unexpected events [7]. Drivers of automated vehicles are also less likely to anticipate possible safety-critical events, resulting in a slower response, even when warned by the system [14]. Such out-of-the-loop behavior carries consequences beyond a transfer to manual control, in which it can take drivers close to a minute to regain stable control of the vehicle following from the takeover point [15]. It is this withdrawal of attention from the driving task (and any consequent re-distribution to nondriving-related activities) that higher levels of automation are prone to induce that is of particular interest in this paper.

To more closely examine driver behavior during semiautonomous control and the shifts in this behavior that result under fully-autonomous control, this study aims to look at voluntary non-driving-related task activity during varying levels of automated control. This study utilized data collected from an experiment, which introduced three different driving modes: (1) manual control mode, (2) semiautonomous vehicle mode, and (3) fully-autonomous vehicle mode. The difference between semi-autonomous vehicle and full-autonomous vehicle modes was simply whether or not a driver was able to initiate lane change decisions (details of each mode are more fully described in the method section). This level distinction does not precisely follow the level taxonomy of automation provided by National Highway Traffic Safety Administration (NHTSA) [16] or the Society of Automotive Engineers [22], However, as implemented, it directly compares the key difference between these two autonomous modes in the expectation of driver monitoring and at a level in which a consumer is capable of fully understating operating characteristics of the systems. In particular, it provides a comparison point on the impact of varying the required amount of interaction with the system, in which the driver would need to actively monitor the driving environment to initiate appropriate lane changes in the semi-autonomous mode but not in the autonomous vehicle mode, since in this mode the vehicle self-initiated lane changes. The central research question is whether or not this subtle/minor difference in drivers' involvement makes any differences in their behavior during highly automated driving. To answer this question, this study analyzed 32 drivers' engagement in non-driving-related activities (as measured by manually annotated hand positions) and gaze-based distraction level (as measured by automatically annotated gaze regions) during three driving modes.

\section{METHOD}

Data for this study was collected as part of collaborative project with Junghee Park; see [19] for detailed consideration of the driving scenario, vehicle metrics, and subjective impressions of participants. Essential elements are presented here. 


\section{Participants}

A total of 36 participants between the ages of 20-29 or 5569 were recruited. (The age split was intended to allow for eventual investigation of whether or not any significant age group related differences might be observed; such a subgroup analysis is not considered in this report.) All reported being in reasonably good health for their age, being fully comfortable speaking and reading English, having a driver's license for over three years, and driving on average at least one day a week. Volunteers were excluded if they had been hospitalized in the past six months, required a pacemaker, or had a diagnosed neurological or psychological disorder. Four participants were excluded from the analysis due to technical issues during the experiment. The final sample consisted of 32 participants balanced equally across age group and gender (Table 1).

\begin{tabular}{l|lll} 
Age group & Female & Male & Combined \\
\hline $\mathbf{2 0 - 2 9}$ & $25.0(3.07)$ & $24.0(2.83)$ & $24.5(2.90)$ \\
$\mathbf{5 5 - 6 9}$ & $60.9(3.94)$ & $62.4(4.63)$ & $61.6(4.22)$
\end{tabular}

Table 1. Mean and standard deviation of age of participants.

\section{Apparatus}

The study was conducted in a full cab fixed-base driving simulator (a 2001 Volkswagen New Beetle). A front projection screen provided an approximate $40^{\circ}$ view of the virtual world. Graphical updates were generated and presented at $50 \mathrm{~Hz}$ using CarSim Driver Simulator version 8.2. The vehicle's sound system was used to present prerecorded instructions and auditory feedback consisting of engine, turn signal, and braking sounds. Two cameras were mounted in the cab, one positioned above the instrument cluster recording the participant's face (observing gaze), the other on the ceiling of the cab recording the steering wheel and the participant's lap (observing interactions with the steering wheel and secondary activities). The cameras recorded at $30 \mathrm{fps}$.

The simulation scenario consisted of a highway with four lanes in the direction of travel. A rearview mirror was projected to show traffic behind the participant vehicle. Lane width was $5.0 \mathrm{~m}$ and the posted speed limit was 55 mph $(88.5 \mathrm{~km} / \mathrm{h})$. Traffic was simulated by placing eight vehicles, two in each lane, on the highway [19].

\section{Assistance Systems}

Three driving modes were implemented and experienced by all participants: manual, semi-autonomous, and fullyautonomous. In the manual mode the participant was in full control of the vehicle (steering, accelerating, and braking). In semi-autonomous mode the vehicle remained in the current lane by steering itself and adjusting its speed to avoid collisions. The participant was able to change lanes by using the turn signal to indicate a direction; the vehicle then changed lanes automatically if it was safe to do so. If it was unsafe to change lanes, the vehicle would remain in the lane and an indicator above the instrument cluster would flash to show the command could not be completed. In fully-autonomous mode the vehicle was in full control, including steering, speed, and lane change decisions. In both autonomous modes, the participant was unable to brake, steer, or resume full control of the vehicle.

\section{Secondary Activity Items (Distractors)}

For the experimental phase of the study (see next section) a number of items were placed on the passenger seat: a newspaper, three magazines, a water bottle, a bag of pretzels, a bag of chips, an energy bar, a pack of gum, and a bag of individually-wrapped candies. The newspaper was current and replaced daily. If a participant opened an item of food it was replaced for the next participant. Additionally, participants were allowed access to their personal cellphones.

\section{Procedure}

Participants were first given an overview of the experiment and an explanation of the three different driving modes. They were told that they would be paid a minimum of US\$50 for their participation and had the opportunity to earn a US\$10 incentive based upon their performance (calculated based on their ability to maintain a speed near to the $55 \mathrm{mph}$ speed limit and to avoid vehicle collisions). In actuality, all participants were paid US\$60 independent of performance.

The study began with a five-minute familiarization drive to habituate the participants with the simulator. Participants were then asked to drive for 10 minutes in each of the three modes (30 minutes total), presented in a random order, as training. After the training period, participants were given a short break before being presented with the following instructions for the experimental portion:

In this half of the study, we would like you to imagine you are going on a long car trip. Just as in real life, you want to get to your destination safely and on time. You may continue driving in one lane, or move into another lane so you are comfortable with the traffic flow. You are welcome to use your cell phone as you imagine you would during such a drive - feel free to call or text a friend, check your email, play music, or whatever other activity you feel comfortable doing in each driving scenario. You've also brought along some magazines, today's paper, and some snacks that will be kept on the passenger's seat. Please help yourself to any of these items at any point while you are driving. You are not obligated to use any of these items, and driving safely as you would in a real car is still your first priority. However, if you imagine you would use these items while driving in real life, please use them today.

The secondary activity items were then placed onto the passenger seat and participants were instructed to turn on their cellphone and bring it with them into the vehicle. Participants then drove in each mode again in randomized 10 minute blocks. 


\section{Manual Coding of Hand Positions}

A single pass of the positions and contents of participants' hands were manually coded from video analysis by two independent coders. No distinction between left and right hands was made for hand position. Items in the participants' laps were not recorded unless they were actively being held by the participant.

\section{Automated Coding of Gaze Region}

Driver gaze was automatically annotated using a gaze classification approach [5,6] as being either "on-road" or "off-road." In this process, semi-frontal face detection is required. Frames for which no semi-frontal face was detected were not annotated as that is a prerequisite for the image processing pipeline.

\section{Data Analysis}

Drivers' gaze, hand position, and the duration of their interaction with each distractor item were analyzed across the three driving modes. First, a main effect of driving mode on percentage of time without engagement with the distractor items was tested by a linear mixed-effect model with one fixed effect (i.e., driving mode) and one random effect (i.e., participant). Second, another linear mixed-effect model with two fixed effects (driving mode and distraction type) and one random effect (i.e., participant) was applied to test the effects on percentage of time spent on distractors. Third, a similar model with driving mode and hand position as fixed effects and participant as a random effect was applied to test effects on percentage of time for each hand position type. Due to unequal variances and unbalanced sample sizes, Welch's t-test was applied for post-hoc tests. Also, marginal $\mathrm{R}^{2}$ (the proportion explained by the fixed factor alone) and conditional $\mathrm{R}^{2}$ (the proportion explained by the fixed and random factors), and Cohen's $\mathrm{d}$ (standardized difference between two means) were reported for effect sizes. Kenward-Roger correction was also applied to adjust the F statistics and degrees of freedom.

\section{RESULTS}

\section{Engagement in Secondary Physical Activities}

Figure 1 presents the percentage of time participants physically engaged in activities secondary to driving such as holding their phone, reading a paper, or handling food during each of the three driving modes. Considering first the binary condition of holding a distractor item vs. not engaging with an item, there was a significant difference across driving modes, $F(2,62)=58.32, p<.001$, marginal $R^{2}=0.42$, and conditional $R^{2}=0.65$. Post-hoc t-tests showed that the manual mode led to a higher percentage of time without engagement with distractors than the semiautonomous mode, $t(41.24)=5.57, p<.001, d=1.39$, and the fully-autonomous mode, $t(42.91)=9.52, p<.001$, $d=2.38$. Likewise, the semiautonomous mode led to a higher percentage of time without distractors than the fullyautonomous mode, $t(61.60)=2.64, p<.05, d=0.66$.

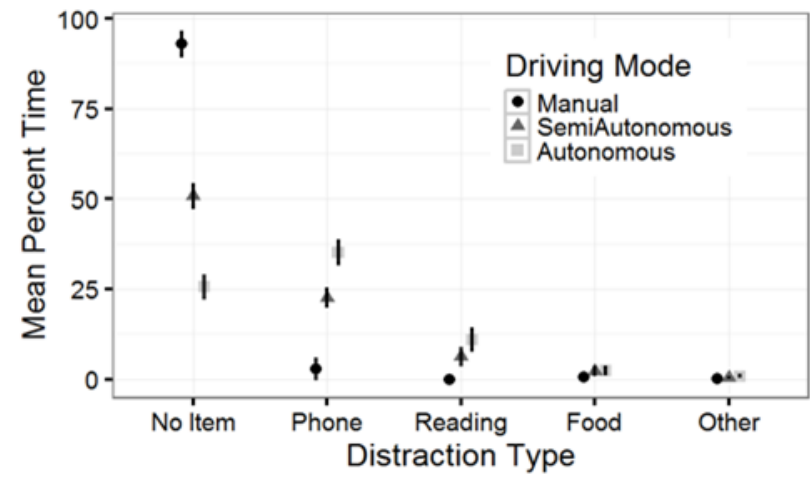

Figure 1. Mean percent and standard error of the time participants held on to each type of distractor in each driving mode. ("No Item" corresponds to no distractor held.)

Second, analyses were run that considered distractor type (phone, reading, food, other) and driving mode (Figure 1). Significant differences appeared across driving mode, $F(2,923)=11.36, p<.001$, and distractor type, $F(3,923)=57.30, p<.001$, marginal $R^{2}=0.17$, and conditional $R^{2}=0.17$. There were significant differences in phone use across driving mode, $F(2,62)=17.16, p<.001$, marginal $R^{2}=0.18$, and conditional $R^{2}=0.51$. Post-hoc t-test showed that the manual mode led to less percentage of time spent using the phone than the semi-autonomous mode, $t(43.61)=-3.29, p<.01, d=-0.82$, and the fullyautonomous mode, $t(40.12)=-4.65, p<.001, d=-1.16$. No significant difference in phone use was found between semi-autonomous and fully-autonomous mode, $t(60.23)=-1.49, p=0.14, d=-0.37$.

\section{Drivers' Hand Positions}

Percentage of time with hands in each position was tested first (both hands on the steering wheel, both hands off the steering wheel and empty, both hands holding a distractor item, one hand on the steering wheel and one hand off but empty, one hand on the steering wheel and the other holding an item, both hands off the steering wheel and an item in one hand, other positions, hands not visible); note that the categories "other hand positions" and "hands not visible" are not shown in Figure 2, but are included in the

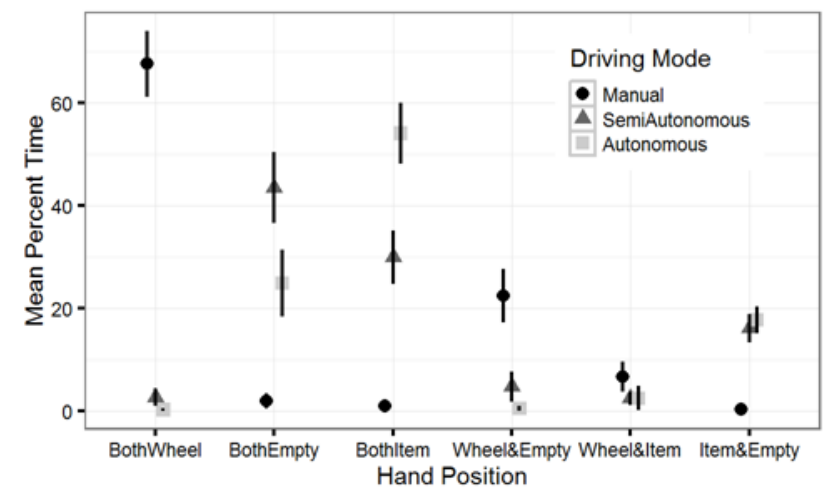

Figure 2. Mean percent of time and standard error participants had their hands in each position by driving mode. 
analysis. There was a significant difference in mean duration by hand position, $F(7,599)=12.20, p<.001$, marginal $\mathrm{R}^{2}=0.12$, and conditional $\mathrm{R}^{2}=0.12$.

There was also a significant difference in mean duration having both hands empty across driving modes, $F(2,62)=$ $17.35, p<.001$, marginal $R^{2}=0.23$, and conditional $R^{2}=0.38$. Post-hoc t-tests showed that the manual mode led to less percentage time with empty hands than the semiautonomous mode, $t(33.71)=-5.84, p<.001, d=-1.46$, and fully-autonomous mode, $t(34.08)=-3.42, p<.01$, $d=-0.84$. No significant difference for empty hands was found between semi- and fully-autonomous modes, $t(61.74)=1.96, p=0.06, d=0.49$.

Percentage of time with both hands holding an item was tested. There was a significant difference across driving modes, $F(2,62)=45.01, p<.001$, marginal $R^{2}=0.41$, and conditional $R^{2}=0.56$. Post-hoc t-tests showed that manual mode led to less percentage time with both hands on an item than semi-autonomous mode, $t(32.21)=-5.54$, $p<.001, d=-1.46$, and fully-autonomous mode, $t(31.91)=$ $-8.83, p<.001, d=-2.21$. Likewise semi-autonomous mode led to less percentage of time with both hands on an item than fully-autonomous mode, $t(60.79)=-3.06, p<.01$, $d=-0.76$.

Percentage of time with at least one hand on the wheel was calculated by summing "BothWheel", "Wheel\&Empty", and "Wheel\&Item" for each participant. There was a significant difference between driving modes, $F(2,62)=$ $327.18, p<.001$, marginal $R^{2}=0.84$, and conditional $R^{2}=$ 0.88 . Post-hoc t-tests showed that the manual mode led to more wheel holding than the semi-autonomous mode, $t(49.39)=17.86, p<.001, d=4.46$ and the fullyautonomous mode, $t(59.51)=24.40, p<.001, d=6.1$. No significant difference between semi- and fully-autonomous modes, $t(55.73)=1.30, p=0.20, d=0.32$ was found.

Percentage of time with at least one hand on a distractor was calculated by summing "BothItem", "Item\&Empty", and "Wheel\&Item" for each participant. There was a significant difference between driving modes, $F(2,62)=$ $57.80, p<.001$, marginal $R^{2}=0.41$, and conditional $R^{2}=$ 0.66. Post-hoc t-tests showed that manual mode led to less distractor holding than the semi-autonomous mode, $t(42.00)=-5.32, p<.001, d=-1.31$, and fully-autonomous mode, $t$ (43.79) $=-9.31, p<.001, d=-2.33$. Likewise, semi-autonomous mode led to less distractor holding than fully-autonomous mode, $t(61.59)=-2.70, p<.01$, $d=-0.68$.

The time before a participant picked up a first distractor item after the beginning of a driving period is shown in Figure 3. Some participants began a drive already holding a distractor item; these were included as 0 seconds.

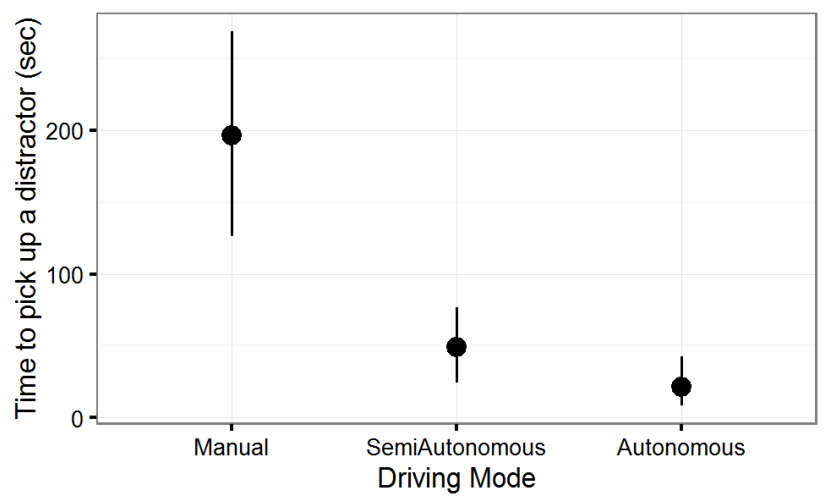

Figure 3. Mean time and standard deviation in seconds until participants picked-up a distractor item after the start of each driving mode.

\section{Driver Gaze}

Percentage of time that each driver spent looking away from the forward roadway (Figure 4) was computed in an automated way using gaze classification methods developed in $[5,6]$. The main steps of this process are (1) face detection, (2) face alignment, (3) pupil detection, (4) head and eye pose estimation, and (5) binary classification into one of two gaze regions: on-road and off-road. Dlib's histogram-of-oriented-gradient (HOG) based object detector [11] was used to train a semi-frontal face detector. The visibility of the face is a prerequisite for successful classification, and because of this the percentage of video frames with a successfully detected face serves as metric for the frequency of drastic head movements away from the frontal position. The first numeric column of Table 2 shows that face detection becomes progressively more difficult with increasing levels of automation and the second numeric column shows that when a semi-frontal face is successfully detected, the percentage of time that the driver's gaze is directed off-road increases with increasing levels of automation.

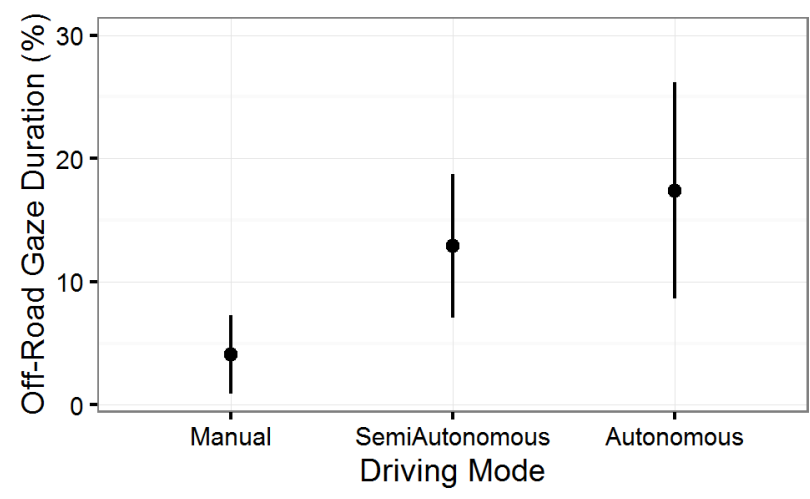

Figure 4. Percentage of time with gaze directed off-road for each of the three driving modes. Error bars indicate interparticipant standard deviation. 


\begin{tabular}{|l|cc} 
Driving mode & $\begin{array}{c}\text { Percentage of } \\
\text { face detections }\end{array}$ & $\begin{array}{c}\text { Percentage of duration } \\
\text { with gaze directed off-road }\end{array}$ \\
\hline Manual & $68.8 \%$ & $4.1 \%$ \\
Semiautonomous & $54.8 \%$ & $12.9 \%$ \\
Autonomous & $49.3 \%$ & $17.4 \%$
\end{tabular}

Table 2. Automated gaze classification results showing both increasing face detection difficulty and off-road glance duration with increasing levels of automation.

\section{DISCUSSION}

The results show that during periods of high levels of automation, participants spent more time engaged in objectively visible secondary activities, and with one or more hands off the wheel, as compared to manual driving. While it was expected that drivers in the two autonomous modes (semi-; fully-), as compared to manual mode, would spend more time with their hands off the wheel because steering is system-controlled, of interest is the finding of increased time spent with both hands holding an item unrelated to the driving task. This tendency to pick-up a secondary object and to engage both hands is more pronounced in fully-autonomous compared to semiautonomous mode. Unsurprisingly, an analysis of the individual items showed that the cell phone was the primary item participants picked-up and interacted with. It is possible that this is due to a preference for a personal item over supplied items, although the increasingly ubiquitous experience with the smartphone as something one can pickup when there is a spare moment to fill might equally likely be proposed as an explanation. The effect of distractor type has on hand usage was not explored; likely the number of hands required to interact with an item differs by type (e.g., newspaper folding probably requires two hands).

These results confirm recent findings that higher levels of automation quickly induce driver comfort and willingness to engage in non-driving related activities [2,9]. This reallocation of attention to non-driving related activities has the potential to counteract many of the safety benefits of automated vehicle technologies to the extent that drivers are needed to provide back-up driving control. What is unclear however, is the degree to which engagement in a secondary activity represents classic "driver distraction" or an understandable need to fill spare attentional capacity (e.g. Yerkes-Dodson) with "something else to do". As such, helping drivers stave-off boredom. In short, secondary activities may be pivotal in the longer term quest to keep drivers alert enough to be able to resume operational control when requested in Levels 2 \& 3 (SAE) automated systems. As automation systems become increasingly capable and reliable, the need for driver alertness should decrease and the overall benefits (safety, mobility, etc.) of autonomous driving will in turn likely increase.

Though these results confirm the previously-reported tendency for drivers to spend more time interacting with non-driving-related items with increased levels of automation, they also hint at the promise of shared control approaches to automation. Shared control approaches are those in which the human maintains some level of control and/or an ability to shift between authority levels $[1,4]$. Simply providing drivers with the ability to change lanes through use of the turn signal - to decide when to initiate the automated system's lateral control functionality-kept them more engaged in the primary driving task; i.e., in the semi-autonomous mode, as a group, drivers in this simulation were less likely to pick up a secondary object, and more likely to keep their hands free or on the wheel. Drivers' involvement in a vehicle's strategic decision making (i.e., lane changes in the case of this study) and action selection activities and/or action implementation as part of mid-levels of automation, may encourage them to stay 'in the loop', supporting their ability to resume manual control if needed [18]. That being said, some of the measured differences between the semi-autonomous and autonomous modes were relatively modest, and the extent to which these will carry-over to real-world driving and conditions requiring extended vigilance need to be better understood.

\section{CONCLUSION}

The results of automated gaze classification revealed two key observations about driver behavior in each of the three driving modes. First, as the level of automation increases, the percentage of time that drive gaze is directed off-road increases. This confirms that the observed increase in nondriving-related task activity is coupled with reduced scanning of the forward roadway. The removal of attention from the forward roadway carries important safety consequences in terms of a driver's ability to anticipate and react to unexpected events outside of the automation's sensing or response capabilities, and to quickly have the awareness needed to resume manual control if requested $[8,15]$. Second, the percentage of time when a semi-frontal face can be detected with a monocular dashboard mounted camera decreases with increasing levels of automation. This is an indicator of more drastic head movements associated with non-driving-related activities. Furthermore, it reveals a fundamental shift in the sensing requirements for robust driver state detection during automated driving. As the driver's eyes, head, and body begin to shift their orientation from being directed towards the forward roadway, effective driver state detection may require camera placements that capture a greater range of gaze and full-body pose variations. Such considerations may call for a greater consideration of broad in-cab sensing to ensure that automated driving systems can accurately estimate the driver's capabilities to resume control if requested. Future work may need to consider decision fusion between external sensing, path planning and operator state such that second-level operational decisions and minute-level tactical maneuvering, and hour-level strategic navigational choices are made based upon driver capacity for involvement. 


\section{LIMITATIONS}

While the automated driving system used in this work encompassed a number of decision features that mimic real world vehicle control, the degree to which operator behaviors observed in a fixed-based driving simulator resemble novice or experienced operators of automated driving systems in real life is unknown. Total exposure to each of the three driving modes (10 minutes familiarization and 10 minutes evaluation - 20 minutes total for each type of driving) was relatively substantive for a laboratory experience, but limited compared to daily exposure. Validation of the findings in this report through naturalistic driving data collection in Level 2 (SAE) vehicles is underway in our laboratory.

\section{ACKNOWEDGEMENT}

Support for this work was provided by the US DOT's Region I New England University Transportation Center at MIT and the Toyota Class Action Settlement Safety Research and Education Program. The views and conclusions being expressed are those of the authors, and have not been sponsored, approved, or endorsed by Toyota or plaintiffs' class counsel.

\section{REFERENCES}

1. David A. Abbink, Mark Mulder, and Erwin R. Boer. 2012. Haptic shared control: Smoothly shifting control authority? Cognition, Technology and Work 14, 1: 1928. http://doi.org/10.1007/s10111-011-0192-5

2. Myra Blanco, Jon Atwood, Holland M. Vasquez, et al. 2015. Human Factors Evaluation of Level 2 and Level 3 Automated Driving Concepts. NHTSA. (Report No. DOT HS 812 182), August: 300. http://doi.org/10.13140/RG.2.1.1874.7361

3. Oliver Carsten, Frank C. H. Lai, Yvonne Barnard, A. Hamish Jamson, and Natasha Merat. 2012. Control Task Substitution in Semiautomated Driving: Does It Matter What Aspects Are Automated? Human Factors 54, 5: 747-761. http://doi.org/10.1177/0018720812460246

4. Frank Ole Flemisch, Klaus Bengler, Heiner Bubb, Hermann Winner, and Ralph Bruder. 2014. Towards cooperative guidance and control of highly automated vehicles: H-Mode and Conduct-by-Wire. Ergonomics 57, 3: 343-60. http://doi.org/10.1080/00140139.2013.869355

5. Lex Fridman, Philipp Langhans, Joonbum Lee, and Bryan Reimer. 2016. Driver Gaze Region Estimation Without Using Eye Movement. Intelligent Systems 31, 3: 49-56.

http://doi.org/http://doi.ieeecomputersociety.org/10.110 9/MIS.2016.47

6. Lex Fridman, Joonbum Lee, Bryan Reimer, and Trent Victor. 2016. "Owl" and "Lizard": Patterns of Head Pose and Eye Pose in Driver Gaze Classification. IET Computer Vision. http://doi.org/10.1049/ietcvi.2015.0296
7. Christian Gold, Daniel Damböck, L. Lorenz, and K. Bengler. 2013. "Take over!" How long does it take to get the driver back into the loop? Human Factors 57, 1: 1938-1942. http://doi.org/10.1177/1541931213571433

8. Christian Gold, Daniel Damböck, L. Lorenz, and K. Bengler. 2013. "Take over!" How long does it take to get the driver back into the loop? Proceedings of the Human Factors and Ergonomics Society Annual Meeting 57, 1: 1938-1942. http://doi.org/10.1177/1541931213571433

9. A. Hamish Jamson, Natasha Merat, Oliver M J Carsten, and Frank C H Lai. 2013. Behavioural changes in drivers experiencing highly-automated vehicle control in varying traffic conditions. Transportation Research Part C: Emerging Technologies 30: 116-125. http://doi.org/10.1016/j.trc.2013.02.008

10. Colin J Kessel, Christopher D Wickens, and Movable Shelves. 1982. Transfer of failure-detection skills between monitoring and controlling. Human Factors 24, 1: 49-60. http://doi.org/10.1177/001872088202400106

11. Davis. E. King. 2009. Dlib-ml: A Machine Learning Toolkit. Journal of Machine Learning Research 10: 1755-1758. http://doi.org/10.1145/1577069.1755843

12. Norman H. Mackworth. 1948. The breakdown of vigilance durning prolonged visual search. Quarterly Journal of Experimental Psychology 1, 1: 6-21. http://doi.org/10.1080/17470214808416738

13. Lucas Malta, Mikael Ljung Aust, Freek Faber, et al. 2012. EuroFOT final results: impacts on traffic safety. Brussels.

14. Natasha Merat and a. H. Jamson. 2008. How do drivers behave in a highly automated car? Proceedings of the Fifth International Driving Symposium on Human Factors in Driver Assessment, Training and Vehicle Design HOW: 514-521.

15. Natasha Merat, A. Hamish Jamson, Frank C H Lai, Michael Daly, and Oliver M J Carsten. 2014. Transition to manual: Driver behaviour when resuming control from a highly automated vehicle. Transportation Research Part F: Traffic Psychology and Behaviour 27, PB: 274-282. http://doi.org/10.1016/j.trf.2014.09.005

16. NHTSA. 2016. 2016 Update to "Preliminary Statement Of Policy Concerning Automated Vehicles." NHTSA. http://doi.org/10.1017/CBO9781107415324.004

17. Donald A Norman. 1990. The "problem" with automation: inappropriate feedback and interaction, not "over-automation". Philosophical Transactions of the Royal Society of London. Series B, Biological sciences 327, 1241: 585-93. http://doi.org/10.1098/rstb.1990.0101

18. Raja Parasuraman, Thomas B Sheridan, and Christopher D Wickens. 2000. A model for types and levels of human interaction with automation. IEEE transactions 
on systems, man, and cybernetics. Part A, Systems and humans : a publication of the IEEE Systems, Man, and Cybernetics Society 30, 3: 286-97. http://doi.org/10.1109/3468.844354

19. Junghee Park, Bryan Reimer, and Karl Iagnemma. A User Study of Semi-Autonomous and Autonomous Highway Driving: An Interactive Simulated Environment. IEEE Intelligent Transportation Systems Magazine: 1-10.

20. Bryan Reimer. 2014. Driver Assistance Systems and the Transition to Automated Vehicles: A Path to Increase Older Adult Safety and Mobility? Public Policy \& Aging Report 24, 1: 27-31. http://doi.org/10.1093/ppar/prt006

21. Bryan Reimer, Bruce Mehler, Jonathan Dobres, and Joseph F Coughlin. 2015. Technical Report 2015-15 Phase II Experiment 4 - An Exploratory Study of Driver Behavior With and Without Assistive Cruise Control ( $A C C$ ). Cambridge, MA. http://doi.org/10.13140/RG.2.1.3817.2565

22. SAE International. 2014. Taxonomy and Definitions for Terms Related to On-Road Motor Vehicle Automated Driving Systems. SAE International J3016: 1-12.

23. Mehdi Saffarian, Joost C. F. de Winter, and Riender Happee. 2012. Automated Driving: Human-Factors Issues and Design Solutions. Proceedings of the Human Factors and Ergonomics Society Annual Meeting 56, 1: 2296-2300. http://doi.org/10.1177/1071181312561483

24. Jeremy Salinger. 2012. Human Factors for LimitedAbility Autonomous Driving Systems. Retrieved from http://onlinepubs.trb.org/onlinepubs/conferences/2012/A utomation/presentations/Salinger.pdf

25. Nadine B. Sarter, David D. Woods, and Charles E. Billings. 1997. Automation Surprises. Human Factors: $1-25$.

26. David Schrank., Bill Eisele, Tim Lomax., and Jim Bak. 2015. 2015 Urban Mobility Scorecard. Texas A\&M Transportation Institue \& INRIX Inc., August 2015.

27. Judi E. See, Steven R. Howe, Joel S. Warm, and William N. Dember. 1995. Meta-analysis of the sensitivity decrement in vigilance. Psychological Bulletin 117, 2: 230-249. http://doi.org/10.1037/00332909.117.2.230

28. Bobbie D. Seppelt and T.W. Victor. Potential solutions to human factors challenges in road vehicle automation. In Road Vehicle Automation 3. Springer Book Series.

29. Neville A. Stanton and Mark S. Young. 1998. Vehicle automation and driving performance. Ergonomics 41, 7: 1014-1028. http://doi.org/10.1080/001401398186568

30. Emma Tivesten, Alberto Morando, and Trent Victor. 2015. The Timecourse of Driver Visual Attention in Naturalistic Driving With Adaptive Cruise Control and Forward Collision Warning the Timecourse of Driver
Visual Attention in Naturalistic Driving With Adaptive Cruise. Conference on Driver Distraction and Inattention, 1-14.

31. Joel S. Warm (ed.). 1995. Sustained attention in human performance. Wiley, Chichester.

32. Christopher D. Wickens. 1992. Engineering psychology and human performance. Harper Collins, New York. 